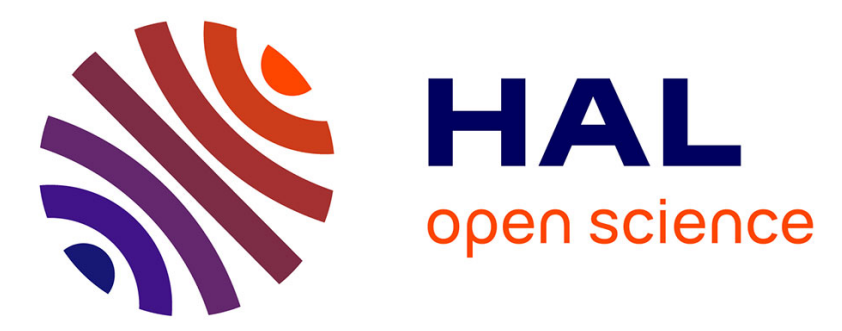

\title{
La diffusion isiaque en Mésie Inférieure et en Thrace: politique, commerce et religion
}

Laurent Bricault

\section{To cite this version:}

Laurent Bricault. La diffusion isiaque en Mésie Inférieure et en Thrace: politique, commerce et religion. Proceedings of the IIIrd International Conference of Isis Studies, Faculty of Archaeology, Leiden University, May 11-14 2005, 2007, Leiden, Pays-Bas. pp.245 - 266. hal-01817120

\section{HAL Id: hal-01817120 \\ https://hal.science/hal-01817120}

Submitted on 18 Jun 2018

HAL is a multi-disciplinary open access archive for the deposit and dissemination of scientific research documents, whether they are published or not. The documents may come from teaching and research institutions in France or abroad, or from public or private research centers.
L'archive ouverte pluridisciplinaire HAL, est destinée au dépôt et à la diffusion de documents scientifiques de niveau recherche, publiés ou non, émanant des établissements d'enseignement et de recherche français ou étrangers, des laboratoires publics ou privés. 


\section{PART TWO}

UNDERSTANDING THE CULTS OF ISIS

IN THEIR LOCAL CONTEXT 

THE BALKANS \& GREEGE 



\title{
LA DIFFUSION ISIAQUE EN MÉSIE INFÉRIEURE ET EN THRACE: POLITIQUE, COMMERCE ET RELIGION
}

\author{
Laurent Bricault
}

Une enquête sur les cultes isiaques en Mésie Inférieure et en Thrace avait été proposée il y a une vingtaine d'années par M. TachevaHitova dans le corps de son ouvrage Eastern Cults in Moesia inferior and Thracia ${ }^{1}$. Il a semblé toutefois qu'un certain nombre d'éléments pouvaient être précisés, sinon amendés, et quelques autres documents ajoutés au dossier, permettant de proposer une vision actualisée de la diffusion isiaque dans ces régions. ${ }^{2}$

Les éléments dont nous disposons aujourd'hui montrent que les cultes isiaques se sont implantés dans les ports du littoral européen du Pont-Euxin, de la Propontide et de la Mer de Thrace dès le III ${ }^{e}$

1 Tacheva-Hitova, Eastern Cults 3-67 et notes 280-285. Plus anciens sont les ouvrages de Drexler, Cultus 59-89 et 97-122, et Rusch, De Serapide. On pourra également consulter Dunand, Culte d'Isis 63-66 et 198-201 pour la présence isiaque en Thrace, Vidman, Donauprovinzen 998-1008, et M.-Chr. Budischovsky, Témoignages de dévotion isiaque et traces culturelles le long du limes danubien, dans: Isis en Occident 173174 , pour les documents à caractère isiaque de Mésie Inférieure. Pour un aperçu rapide de leur diffusion dans le nord des Balkans, Hölbl, Balkanraum.

${ }^{2}$ La majeure partie des documents relatifs à la diffusion isiaque est réunie dans Bricault, Atlas 28-33. Ils ne seront donc pas tous repris dans cette synthèse, qui apportera toutefois quelques précisions sur certains d'entre eux, notamment grâce à l'obligeance de Marie-Christine Budischovsky, que je tiens à remercier vivement pour m'avoir généreusement transmis certaines de ses fiches. Les sources épigraphiques sur le sujet sont regroupées dans le RICIS, vol. I 176-190 et vol. II 734-742. Les émissions monétaires à types isiaques, très nombreuses pour les cités de Mésie Inférieure et de Thrace, figureront dans une Sylloge Nummorum Religionis Isiacae et Sarapiacae (= SNRIS), répertoire analytique conçu en collaboration internationale, à paraître en 2007. Le commentaire et l'analyse de ces sources très riches mais méconnues, pour les régions qui nous occupent ici, sont l'œuvre d'U. Peter (Berlin). Certains résultats partiels ont déjà été présentés ailleurs par U. Peter, Zur Problematik der Isis- und Sarapistypen in der Münzprägung der westpontischen Städte, dans: Numismatic and Sphragistic Contributions to History of the Western Black Sea Coast, International Conference Varna, septembre 12th-15th, 2001, Acta Musei Varnaensis II (Varna 2004) 175-190; ead., Religious-cultural Identity in Thrace and Moesia Inferior, dans: Chr. Howgego, V. Heuchert, A. Burnett (éds.), Coinage and Identity in the Roman Provinces (Oxford 2005) 107-114; ead., Götter auf Reisen. Münzbilder über die Verbreitung ägyptischer Kulte, dans: Abhandlungen der Berlin-Brandenburgischen Akademie der Wissenschaften. Sonderband (à paraître). 
siècle av. J.-C. Les hypothèses n'ont pas manqué pour expliquer cette diffusion relativement rapide et précoce - même si elle l'est probablement moins qu'on ne l'a longtemps cru- Deux thèses successives et opposées ont longtemps dominé les débats. D'une part, celle de Franz Cumont pour qui les Ptolémées favorisèrent au III siècle av. J.-G., dans leur sphère d'influence, le développement du culte de Sarapis, dieu portant la marque de la souveraineté lagide, et des principales divinités de son cercle, c'est-à-dire Isis, Anubis et Harpocrate, et d'autre part celle défendue en premier par Peter Marshall Fraser, qui considérait qu'on ne pouvait en aucun cas attribuer la diffusion de ces cultes à une influence politique ptolémaïque directe. Pendant de nombreuses années, les modernes ont cherché à défendre l'une ou l'autre de ces deux thèses, a priori opposées, sur les vecteurs humains de la diffusion isiaque, les uns affirmant l'attraction exercée sur les Grecs par la fascinante religion de l'ancienne Égypte, les autres défendant l'idée d'une «Isispropaganda » orchestrée par le clergé égyptien, l'une ou l'autre thèse étant censée répondre à tous les cas de figure. L'étude de la situation présentée par les régions qui nous occupent ici montre que l'on ne peut proposer une réponse unique, mais que les éléments à prendre en considération sont multiples et variés, déterminant - en exagérant un peu - presque autant de cas particuliers qu'il $\mathrm{y}$ a de cas de figure.

Tout d'abord, il me semble que l'on doit distinguer le cas d'Isis de celui de Sarapis, au moins jusqu'au règne de Ptolémée Évergète. Les plus anciens documents provenant de nos régions mentionnant Isis sont une dédicace de Périnthe adressée à Isis-Aphrodite par l'un de ses prêtres, que je daterais du milieu voire du $3^{\mathrm{e}}$ quart du $\mathrm{III}^{\mathrm{e}}$ siècle av. J.-C. ${ }^{3}$ une autre dédicace à Sarapis, Isis et Anubis, de Chersonèse en Crimée, gravée sur un autel trouvé en 1993, que les éditeurs, avec vraisemblance, dateraient du milieu du III ${ }^{\mathrm{e}}$ siècle av. J.-C., ${ }^{4}$ et la désormais fameuse fresque polychrome découverte dans une chapelle d'Aphrodite située près du port de Nymphaion, toujours en Crimée,

${ }^{3}$ RICIS 114/0601 = E. Kalinka, 7ÖAI 12 (1926) Beibl. 193 n $152=$ SIRIS n ${ }^{\circ}$ $128=$ Tacheva-Hitova, Eastern Cults n ${ }^{\circ}$ I.47 27-28 = M. H. Sayar, Perinthos-Herakleia (Marmara Ereğlisi) und Umgebung. Geschichte, Testimonien, griechische und lateinische Inschriften (Wien 1998) $225 n^{\circ}$ 42. L'inscription est datée des IV ${ }^{\mathrm{e}}$-III ${ }^{\mathrm{e}}$ siècles av.J.-C. par Kalinka, Fraser et Vidman; de la seconde moitié du $\mathrm{III}^{\mathrm{e}}$ siècle av. J.-C. par Tacheva; des $\mathrm{III}^{\mathrm{e}}$-II ${ }^{\mathrm{e}}$ siècles av. J.-C. par Sayar.

${ }^{4}$ RICIS 115/0302 = Vinogradov/Zolotarev, Egyptian Triad 358-364. 
présentant plusieurs dessins de navires et des inscriptions en relation avec la mer, écrites en grec. ${ }^{5}$ Sur l'un des navires apparaît le nom $\mathrm{I} \Sigma \mathrm{I} \Sigma$. L'identification de ce navire, la signification de ce nom, le sens à donner à cet ensemble pictural et sa datation ont suscité nombre d'interrogations et d'hypothèses que je discute ailleurs. ${ }^{6}$ Ces documents, et bien d'autres, m’ont amené à considérer que la diffusion du culte d'Isis en Méditerranée orientale s'est effectuée simultanément, sinon conjointement à celle du culte rendu à Arsinoé II divinisée, en tant que patronne de la flotte lagide, puis bientôt comme protectrice de tous les marins et de tous les navires. On peut alors supposer que la dédicace de Périnthe, cité dont les Lagides prirent possession en 245 av. J.-C., s'adresse à une Isis-Aphrodite Euploia, ${ }^{7}$ et qu'à Nymphaion la présence dans un temple d'Aphrodite d'une fresque marine, probablement relative à une ambassade lagide, n'est pas fortuite.

La dédicace de Chersonèse s'adresse, outre Isis, à Sarapis et à Anubis. ${ }^{8}$ Associant Sarapis et Isis, un Ptolémée-Philadelphe ou

${ }^{5}$ Cette découverte exceptionnelle, faite en 1982 par une expédition du Musée de l'Ermitage, a suscité une abondante littérature. Outre les nombreuses publications de la directrice des fouilles, N. L. Grač, Otkrytije novogo istoričeskogo istočnika v Nimfee (La découverte d'un nouveau document historique à Nymphaion), VDI (1984/1) 81-88; ead., Ein neu entdecktes Fresko aus hellenistischer Zeit in Nymphaion bei Kertsch, dans: Skythika, Bayerische Akademie der Wissenschaften, Abh. Heft 98 (München 1987) 90-95 et pll. 35-39; ead., K voprosu o političeskich kontaktach Bospora s Egiptom v III v. do n.e. (Sur la question des contacts politiques entre le Bosphore et l'Égypte au IIIe s. av. notre ère, dans Skifija $i$ Bospor (Novočerkassk 1989) 44 sq [en russe, non vidi], on verra également les études de L. Basch, The Isis of Ptolemy II Philadelphus, Mariner's Mirror 71.2 (1985) 129-151; J. S. Morrison, Greek and Roman Oared Warships, Oxbow Monograph 62 (Oxford 1996) 207-214 n 13; Ju. G. Vinogradov, Der Staatsbesuch der "Isis" im Bosporus, Ancient Civilizations from Scythia to Siberia 5.4 (1999) 271-302; O. Höckmann, Naval and other Graffiti from Nymphaion, ACSS 5.4 (1999) 303-356; Vinogradov/Zolotarev, Egyptian Triad. Cf. enfin les deux études de W. M. Murray, A trireme named Isis: the sgraffito from Nymphaion, International Fournal of nautical Archaeology 30.2 (2001) 250-256, et Observations on the 'Isis' Fresco at Nymphaion, dans: H. Tzalas (éd.), Tropis VII, Proceedings of the 7th International Symposium on Ship Construction in Antiquity, Pylos 1999 (Athens 2002) 539-561. Je tiens à remercier le Professeur William Murray pour m'avoir fort aimablement transmis le texte manuscrit de sa communication de 1999, ainsi que d'excellentes photographies de la fresque.

6 Dans un ouvrage à paraître intitulé Isis, Dame des flots.

7 Vidman, Isis und Sarapis 32-34, envisageait déjà un lien entre cette prêtrise et le culte d'Arsinoé II.

${ }^{8}$ Pour les éditeurs, cette inscription se rapporterait à l'introduction des cultes isiaques à Chersonèse par un membre de l'aristocratie locale. C'est à mon sens aller 
Évergète plutôt que Sôter -, au milieu du III siècle av. J.-C., a cherché à mettre en place comme dyade poliade d'Alexandrie un nouveau couple divin, modèle et garant de son pouvoir royal, auquel Anubis est ensuite adjoint, en dehors d'Alexandrie, pour former une triade. Sarapis et Isis, en bustes accolés, apparaissent de fait au droit de monnaies de Périnthe dès la fin du III $^{\mathrm{e}}$ siècle (fig. 1), à l'instar des tétradrachmes de Ptolémée IV figurant le couple divin. ${ }^{9}$ Au revers de ces émissions de la cité thrace, on trouve les images d'Anubis ou d'Apis. L'impossibilité de dater précisément ces frappes - la présence de monogrammes n'autorisant qu'une chronologie relative approximative dans l'absolu-, ne permet pas de trancher entre deux hypothèses fondamentales. Ces monnaies traduisent-elles la dépendance de la cité envers le pouvoir lagide, ou bien se font-elles l'écho de liens commerciaux privilégiés avec l'Égypte ptolémaïque, soit directement-la présence dans ces régions d'Alexandrins est attestée dès le $\mathrm{III}^{\mathrm{e}}$ siècle - ${ }^{10}$ soit indirectement par l'intermédiaire de Rhodes qui adopta très tôt le culte de Sarapis et qui a certainement servi de relais dans sa diffusion en Méditerranée orientale, en Propontide et dans le Pont-Euxin. La découverte de certaines productions locales d'inspiration égyptienne, comme tel récipient biconique montrant une scène d'offrande à Rê-Horakhty retrouvé à Dionysopolis, ${ }^{11}$ peut ressortir de l'un ou l'autre de ces schémas diffusionnels.

Une inscription de Maronée récemment publiée, elle aussi fragmentaire, pourrait poser une question assez semblable si l'on accepte les restitutions proposées dans le RICIS. ${ }^{12}$ Elle est gravée sur la base

\footnotetext{
un peu trop loin dans l'interprétation de cet important document.

${ }^{9}$ L. Bricault, Isis et Sarapis sauveurs de Ptolémée IV à Raphia, Chronique d’Égypte LXXIV 148 (1999) 335-336.

10 À Callatis, la présence d'Alexandrins est attestée dès le III ${ }^{\mathrm{e}}$ siècle av. J.-C. (J. et L. Robert, Bull. [1939] 235), tandis qu'un adorateur des dieux égyptiens originaire de cette ville est connu vers 200 av. J.-C. à Délos (IG XI, 4, 1238 = RICIS 202/0179). Cf. les remarques de Tacheva-Hitova, Eastern cults 54-58, sur le rôle des marchands dans la propagation initiale des cultes isiaques.

11 G. Strauss et G. Tončeva, Ein ägyptisches Motiv an der Schwarzmerküste, GM 47 (1981) 29-41. Plus généralement, A. Barnea, Pour un répertoire commenté des découvertes égyptiennes de Roumanie. Le tombeau de Tomis, Revue Roumaine d'Égyptologie 1 (1997) 13-19.

12 RICIS*114/0207 = D. Triantaphyllos, AD 48 B2 (1993) [1998] 405 (en majuscules, sans séparation des mots) = SEG XLVII (1997) 1068.
} 


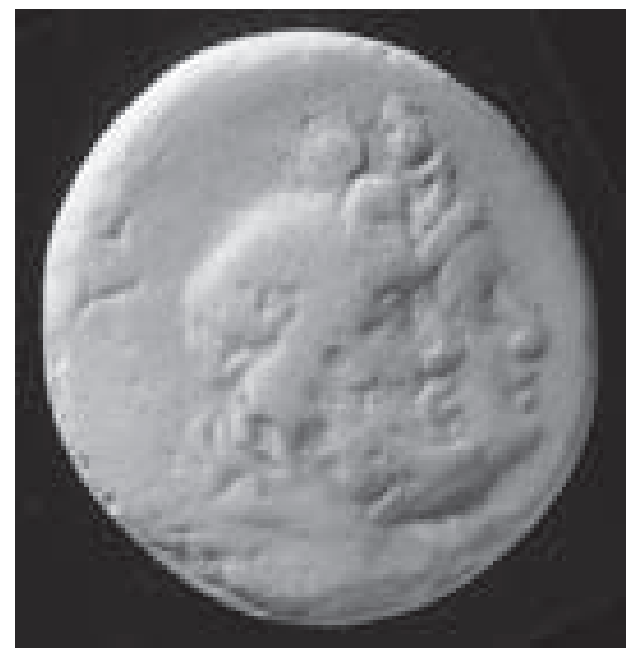

Fig. 1. Monnaie de Périnthe. IIIe s. av. J.-C. (d'après E. Schönert, Perinthos, 34).

d'une statue figurant un roi Ptolémée (peut-être Philométor) et une reine Cléopâtre, dédiée à la suite d'un songe sans doute à Sarapis, Isis et Anubis (mais seules les trois premières lettres du nom de Sérapis sont conservées sur la pierre). L’identité du dédicant ne nous est pas connue. Rappelons que les Ptolémées ont contrôlé Maronée durant la majeure partie du $\mathrm{III}^{\mathrm{e}}$ siècle av. J.-C. ${ }^{13}$, et qu'un lien entre le culte des souverains lagides et celui de la triade isiaque a pu exister dès cette époque, comme on peut l'observer ailleurs, à Théra notamment. ${ }^{14}$

Un décret d'Istros publié en 1964 par Dionys Pippidi pose un autre problème. ${ }^{15} \mathrm{Ce}$ texte, hélas très mutilé, que l'on date volontiers du

13 A. Jähne, Maroneia unter ptolemäischer Herrschaft, dans: U. Peter (éd.), Stephanos nomismatikos. Edith Schönert-Geiss zum 65. Geburtstag (Berlin 1998) 301-316.

14 RICIS 202/1201-1202 et 1204-1205.

15 RICIS 618/1101 = D. M. Pippidi, Sur la diffusion des cultes égyptiens en Scythie Mineure, StudClas VI (1964) 108-118 = SIRIS 709a = Tacheva-Hitova, Eastern Cults 15 n I.22 = ISM I 5 = Vinogradov/Zolotarev, Egyptian Triad 373-376. L'étude de D. M. Pippidi a été rééditée avec quelques compléments et une mise à jour de la bibliographie dans Scythia Minora. Recherches sur les colonies grecques du littoral roumain de la mer Noire (Bucarest 1975) 96-110, et plus récemment dans la Revue Roumaine d'Égyptologie I (1997) 67-86. 
milieu du III ${ }^{\mathrm{e}}$ siècle av. J.-C., rapporte que les Istriens, prolongeant une initiative privée, ont décidé d'envoyer des théores à l'oracle d'Apollon Chresterios, à Chalcédoine, pour l'interroger sur l'opportunité d'introduire un culte officiel de Sarapis à Istros. Malheureusement le texte s'arrête là et l'on ne peut que conjecturer la suite des événements. De l'absence a priori totale d'aegyptiaca provenant d'Istros on pourrait déduire, comme certains l'ont fait, quoique avec une grande prudence, que la réponse de l'oracle fut négative. Mais on ne voit pas alors pourquoi avoir fait graver ce texte. À l'évidence, il le fut a posteriori, une fois la réponse de l'oracle, sans aucun doute positive, connue. La présence de Sarapis cavalier sur des monnaies de la cité aux époques antonine et sévérienne me paraît confirmer cette réflexion, même si les quatre siècles séparant ces frappes du décret invitent à la mesure. Quoi qu'il en soit, Istros ne subit à aucun moment de son histoire l'autorité ou même l'influence politique des Ptolémées. On peut donc estimer que, dans le cas présent, sous l'influence d'Alexandrins présents à Istros ou simplement de personnes ayant intérêt à entretenir de bons rapports avec eux (des Istriens, des Rhodiens?), une démarche officielle fut entreprise pour fonder éventuellement un temple public de Sarapis dans la cité.

Si l'on ignore ce qu'il advint de la démarche des Istriens, d'autres durent aboutir, la présence de sanctuaires isiaques étant attestée avec certitude à Thasos, ${ }^{16}$ Maronée,${ }^{17}$ Périnthe,${ }^{18}$ Chrysorhoas, ${ }^{19}$ Dionysopolis ${ }^{20}$ et Mesembria, ${ }^{21}$ ainsi, selon toutes probabilités, qu'à

${ }^{16}$ RICIS 201/0101 = H. Seyrig, Quatre cultes de Thasos. IV Les dieux égyptiens, BCH 51 (1927) 219-233 = IG XII Suppl. $365=$ F. Sokolowski, Lois sacrées des cités grecques, Supplément (Paris 1962) 130-132 nº 71 = SIRIS 265, à propos de la mise à prix de l'éponymie d'une association de Sarapiastes.

17 RICIS $114 / 0201$ et $114 / 0202$ = Grandjean, Maronée $118-119$ et 17-21 respectivement $=$ Tacheva-Hitova, Eastern Cults 28-31 n I.49-50.

18 Cf. supra 247-248.

19 Un temple de Sarapis y est mentionné par Denys de Byzance (FHG II, 56) et Polybe (IV, 39, 6). Il se trouvait près du Bosphore, à une douzaine de stades de l'entrée de celui-ci, du côté du Pont-Euxin.

${ }^{20}$ RICIS 618/0801 = IGRR I $662=\operatorname{SIRIS~} 703=\operatorname{IGBulg} \mathrm{I}^{2}(1970) \mathrm{n}^{\circ} 13=$ Tacheva Hitova, Eastern Cults 6-7 n I.9. L'inscription, qui mentionne un prêtre de Sarapis qui est aussi prêtre du Grand Dieu, est datée $c a 48$ av. J.-C.

${ }^{21}$ RICIS 114/1401-1404 = Tacheva-Hitova, Eastern Cults 22-27 nº I.42-45. Cf. V. Velkov, Zum kult der ägyptischen Gottheiten in Mesambria Pontica (2-1 Jh.), dans: Hommages à M. F. Vermaseren, ÉPRO 68.III (Leyde 1978) 1293-1295. 
Rhaedestum, ${ }^{22}$ Anchialos, ${ }^{23}$ Tomis, ${ }^{24}$ Tyras ${ }^{25}$ et Chersonèse ${ }^{26}$ aux $\mathrm{II}^{\mathrm{e}}$ et/ou I ${ }^{\mathrm{er}}$ s. av. J.-C. ${ }^{27}$ Les premiers adeptes semblent avoir été des marins et des commerçants, comme cet homme de Sinope qui fait une dédicace $\kappa \alpha \tau \grave{\alpha}$ òv $\alpha \rho$ à Sarapis ${ }^{28}$ parfois constitués en collèges. En 160 apr. J.-C. encore, la guilde des Alexandrins de Tomis se place sous l'invocation de Sarapis. ${ }^{29}$ Certains éléments de la population locale ont également adhéré au culte d'Isis (et de Sarapis) dès le II ${ }^{\mathrm{e}}$ siècle av. J.-C., sinon avant. C'est ce que montre indirectement le texte arétalogique découvert à Maronée et rédigé vers 120 av. J.-C. par un Grec. Les éléments par trop égyptiens de l'arétalogie memphite, qui risquaient d'être incompris des Grecs, ont été purement et simplement supprimés: Osiris a cédé la place à Sarapis - qui n’y est toutefois qu'un simple sunoikos-, les allusions à Sothis, à Bubastis ont disparu, l'Égypte n'est plus qu'un simple lieu de séjour pour une Isis conçue comme une divinité grecque qui aurait quitté Éleusis pour la vallée du Nil, où des colons grecs l'auraient retrouvée. ${ }^{30}$ De fait, la volonté d'hellénisation de la déesse est telle que l'on peut considérer cet éloge comme une véritable interpretatio graeca du texte arétalogique

${ }^{22}$ RICIS 114/0501 = G. Seure, BCH 24 (1900) $164 \mathrm{n}^{\circ} 1$ = E. Kalinka, $\mathcal{F O A I}$ 12 (1926) Beibl. 156 n 3 = SIRIS 127 = Tacheva-Hitova, Eastern Cults 28 n ${ }^{\circ}$ I.48.

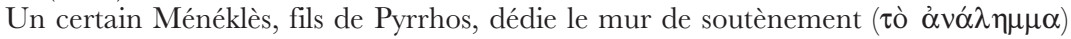
(d'un cadran solaire?) à Sarapis et à Isis. Comparer le cadran solaire en pierre de Durostorum avec les bustes de Sarapis et d'Isis en relief, daté du III ${ }^{\mathrm{e}}$ siècle apr. J.-G. Tacheva-Hitova, Eastern Cults n I. 86, et E. Arslan, Iside n VI.25 566.

${ }^{23}$ RICIS 114/1301 = I. Karayotov, Nouvelles inscriptions de la région de Bourgas (Bulgarie), dans: Actes du VIIè Congrès International d'Épigraphie Grecque et Latine. Constantza 1977 (Bucarest 1979) 388-389 nº 5, dédicace après une guérison à Sarapis et à Isis du $\mathrm{II}^{\mathrm{e}}$ ou du I ${ }^{\mathrm{er}}$ siècle av. J.-C.

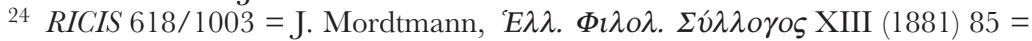
SIRIS 706 = Tacheva-Hitova, Eastern Cults $11 \mathrm{n}^{\circ}$ I.15 = ISM II $181 \mathrm{n}^{\circ} 152$ (37). La dédicace pourrait dater de la fin du $\mathrm{II}^{\mathrm{e}}$ siècle av. J.-C. ou du début du I ${ }^{\mathrm{er}}$ siècle.

${ }^{25}$ RICIS 115/0101 = IOSPE IV, $1=$ IOSPE I I, $5=$ SIRIS 712.

${ }^{26}$ RICIS 115/0302; cf. supra 246.

27 Dans l'Atlas, je mentionnais p. 33, d'après Tacheva-Hitova, Eastern Cults 45, citant Drexler, Cultus 112, des monnaies de Sestos du II siècle av. J.-G. au revers desquelles figurerait le basileion. Ni mes collègues ni moi-même, lors de la préparation de la SNRIS n'avons pu retrouver la trace d'une telle émission, dont l'existence doit être pour le moment mise en doute.

28 RICIS 618/1003.

29 RICIS 618/1005 = IGRR I $604=$ M. Brillant, Inscription de Tomes, RPh 36 (1912) 284-296 = SIRIS 708 = Tacheva-Hitova, Eastern Cults 12-13 n I.17 = ISM II 181-183 n 153 (38).

${ }^{30}$ Outre l'excellente édition du texte par Y. Grandjean, on pourra consulter avec profit F. Mora, Prosopografia Isiaca. II, 47-71. 
originel, sans aucun doute connu de l'auteur, destinée à présenter de la manière la plus compréhensible qui soit la personnalité d'Isis à un public non familier de l'Égypte.

Il est toutefois intéressant de noter que si les documents les plus anciens concernent Isis, si l'arétalogie découverte à Maronée est celle d'Isis « qui a pris Sérapis comme compagnon », si les prêtres nommés le sont souvent de Sarapis et d'Isis, ${ }^{31}$ les temples mentionnés dans les inscriptions d'époque hellénistique sont tous des temples «de Sarapis », à Maronée ${ }^{32}$ comme à Mesembria ${ }^{33}$ et Tomis. ${ }^{34}$ Toutefois, il n'apparaît pas que la popularité de Sarapis soit plus grande que celle de sa compagne. Isis est la plupart du temps mentionnée en compagnie de son parèdre dans les dédicaces hellénistiques, quand elle ne l'est pas seule. ${ }^{35}$ Reconnu pour ses capacités de guérisseur, ${ }^{36}$

31 Ainsi à Tomis (RICIS 618/1004 = IGRR I $621=$ SIRIS $707=$ Tacheva-Hitova, Eastern Cults 12 n I.16 = ISM II 69-70 n 37 [3]), ou à Maronée (RICIS 114/0201).

32 RICIS 114/0203 = D. Triantaphyllos, AD 26 (1971) [1977] 429 (ph. pl. 426); ce décret est considéré erronément comme unpublished par Tacheva-Hitova, Eastern Cults 31 .

${ }^{33}$ RICIS 114/1403 = V. Velkov, Antike Tempel in Mesambria Pontica, 2, Serapistempel in Mesembria, Klio 52 (1970) 468-471 = Tacheva-Hitova, Eastern Cults 26-27 n I.45.

34 RICIS 618/1001 = G. Tocilescu, AEM 6 (1882) $23 \mathrm{n}^{\circ} 46=$ SIRIS $704=$ Tacheva-Hitova, Eastern Cults 8-10 n I.13 = ISM II 42-43 n 7.

35 Ainsi sur ce document peu connu qu'est le bas-relief publié par I. Venedikov dans Nessèbre II (Sofia 1980) $81 \mathrm{sqq}$ (non vidi), et repris par Z. Gočeva, s.v. Hekate (in Thracia), LIMC VI.1 (1992) 1018 n $^{\circ}$ 4. Celui-ci représente une scène de sacrifice, à l'arrière-plan de laquelle se dresse un autel quadrangulaire portant la dédicace "I $\sigma \imath \delta$, autel surmonté d'une statuette d'Hécate à trois visages. Cette plaque de marbre doit dater du $\mathrm{I}^{\mathrm{er}}$ siècle av. J.-C. Ce rapprochement entre Hécate trimorphos et Isis sur la côte orientale du Pont-Euxin se retrouve, me semble-t-il, quelques siècles plus tard, au travers d'une triple dédicace de Jurta, près d'Odessos $($ RICIS $* 618 / 0701=\mathrm{G}$. Tončeva, Musei i pametnici 16.2 [1976] 28-32), dans laquelle Hécate emprunte bien des aspects d'Isis, notamment dans le texte central. Cf. L. Bricault, Isis myrionyme, dans: C. Berger et al. (éds.), Hommages à fean Leclant, 3 (Le Caire 1994) 84-85.

36 RICIS 114/1301, d'Anchialos. Une statuette en bronze d'Isis (et non d'IsisAphrodite comme le suggérait Tacheva-Hitova, Eastern Cults 24), aurait été trouvée près d'une source "guérisseuse ", non loin de la rivière Topolnitsa, à Morozovo (= Chehlare). Cf. Bricault, Atlas 33 (identification à corriger) et le catalogue Le Bronze sculpté de l'époque romaine au Musée archéologique National de Sofia (1984) nº 184 (ph). Une autre statuette d'Isis-Fortuna fut retrouvée dans les bains romains près du village de Pastouša, réputé pour ses sources d'eau. Une photographie de la figurine (aujourd'hui au Musée de Plovdiv, n inv. 389) est donnée par D. Cončev, IAI 17 (1958) 242 fig. 177, ce qui permet de constater qu'il ne s'agit pas de celle représentée sur les pll. XXI de Tacheva-Hitova, Eastern Cults. Cette dernière correspond à une autre statuette en bronze d'Isis-Fortuna, trouvée lors de fouilles menées en 1957-1959 à Madara, à proximité d'une villa romaine. Elle est publiée dans le Bulletin du Musée National šumen I 
le couple divin, et notamment Isis, l'est aussi en tant que protecteur de la navigation. Une dédicace à Sarapis et Isis, provenant de Byzance $^{37}$ et datable du tout début du $\mathrm{I}^{\text {er }}$ siècle apr. J.-C., émane d'un personnage dont l'un des principaux, sinon le principal titre de gloire fut d'avoir été navarque lors des grandes Ploiaphésia, cérémonies marquant la réouverture de la navigation, le 5 mars. Le nom de ces fêtes apparaît également chez Jean le Lydien ${ }^{38}$ et chez Apulée, ${ }^{39}$ qui dépeint avec force le déroulement de la cérémonie. Elles correspondent au Navigium Isidis des calendriers de l'époque impériale. ${ }^{40}$ À Tomis, au III ${ }^{\mathrm{e}}$ siècle apr. J.-C. encore, des hiéronautes réunis en collège sont mentionnés dans une inscription en compagnie de pastophores. ${ }^{41}$ Il est probable que ces fidèles sont à associer d'une façon ou d'une autre à Isis marine.

Par ailleurs, je ne crois pas, contrairement à ce que proposait D. Pippidi, ${ }^{42}$ que l'inscription tomitaine mentionnant au tournant de l'ère chrétienne la fête des X $\alpha \rho \mu$ ó $\sigma v v \alpha$ et, peut-être, le nom d'Isis, soit un document privé émanant d'une association de Sarapiastes. ${ }^{43}$

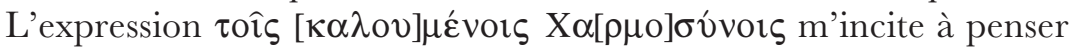
que le nom de cette fête ne devait pas être familier aux lecteurs de la stèle, ce qui étonnerait s'il s'agissait simplement des membres res-

(1960) 51 fig. 52 (renseignements M.-Chr. Budischovsky). Voir aussi la statuette d'Isis provenant des thermes d'Odessos, infra 261 n. 71.

37 RICIS 114/0703 = Th. Wiegand, AthMitt 36 (1911) 287-288 n² $2=$ L. Deubner, AthMitt 37 (1912) 180-182 = L. Robert, Dédicaces aux divinités égyptiennes, Hellenica X (1955) 24-26 = SIRIS 130 = Tacheva-Hitova, Eastern Cults 32-35 n I.54 = I.Byzantion 324. Tacheva-Hitova, Eastern Cults 33-34, propose sans convaincre de rattacher cette inscription à Périnthe ou à Mesembria plutôt qu'à Byzance, et de la dater du règne de Rhoimetalkès III, entre 37 et 45 apr. J.-G., plutôt que du règne de Rhoimetalkès Ier, roi des Odryses, mort en 14 apr. J.-C., qui étendit son pouvoir sur Byzance et Chalcédoine à la fin de son règne. La seule véritable incertitude réside dans le fait de savoir si l'an 32 mentionné dans l'inscription est celui de l'ère d'Actium (soit 1/2 apr. J.-C.), ou correspond à la $32^{\mathrm{e}}$ année du règne de Rhoimetalkès.

38 De mens. IV, 45.

39 Meta. XI, 16-17.

${ }^{40}$ L'expression se lit chez Lactance, Div. Inst. I XI, 21, dans les Ménologes rustiques (RICIS 501/0219) et le «Calendrier de Philocalus» (RICIS 501/0221); sur cette fête, voir F. Perpillou-Thomas, Fêtes d'Égypte ptolémä̈que et romaine, Studia Hellenistica 31 (Louvain 1993) 114-116, et notre étude à paraître sur Isis, Dame des flots.

${ }^{41}$ RICIS 618/1007 = D. M. Teodorescu, Monumente inedite din Tomi (Bucarest 1918) 8-15 n० 3 = SIRIS $709=$ Tacheva-Hitova, Eastern Cults $13-14$ n $^{\circ} \mathrm{I} .18=$ ISM II 123-125 n० 98 (64).

42 D. M. Pippidi, StudClas 6 (1964) 107.

${ }^{43}$ RICIS 618/1001 = G. Tocilescu, AEM 6 (1882) $23 \mathrm{n}^{\circ} 46=$ SIRIS $704=$ Tacheva-Hitova, Eastern Cults 8-10 n I.13 = ISM II 42-43 n 7. 
treints d'une association privée isiaque. Il doit plutôt s'agir d'un décret public. ${ }^{44}$ La fête dont il est question ici est connue par Hérodote, Plutarque, une notice d'Hésychius et plusieurs papyrus. ${ }^{45} \mathrm{Il}$ s'agit très probablement de festivités célébrant la résurrection d'Osiris, que l'on pourrait rapprocher des Hilaria romaines. ${ }^{46}$

Au terme de ce rapide tour d'horizon hellénistique, force est de constater qu'avant l'époque flavienne, toute la documentation isiaque de ces régions est littorale. A priori, les cultes ne semblent pas s'être propagés dans l'intérieur des terres avant la fin du ${ }^{\text {er }}$ siècle apr. J.-C.

Cette pénétration apparaît comme le fruit de la présence romaine, sinon de la romanisation de la Mésie Inférieure et de la Thrace. Remontant les voies terrestres et fluviales, les cultes isiaques quittent les côtes de la Propontide et du Pont-Euxin pour s'avancer, entre la fin du $\mathrm{I}^{\mathrm{er}}$ et le début du $\mathrm{II}^{\mathrm{e}}$ siècle, dans les territoires en question. Ce mouvement vers le nord et l'ouest doit être à peu près contemporain de celui qui, via la Vénétie et la Pannonie, propage vers l'est le culte des mêmes divinités. En 78 apr. J.-C., ${ }^{47}$ un sanctuaire est dédié aux dieux Augustes Isis et Sérapis ainsi qu'aux dieux qui partagent le même temple ${ }^{48}$ (dont Isis-Tychè) ${ }^{49}$ par le peuple des Neinénoi,

${ }^{44}$ Cf. déjà P. M. Fraser, Two studies on the cult of Sarapis in the hellenistic world, OpAth 3 (1960) 38 n. 2.

${ }^{45}$ Hérodote III, 27 (mais l'expression utilisée, $\chi \alpha \rho \mu$ ó $\sigma v v \alpha \tau \alpha \hat{v} \tau \alpha$, est peut-être générique et ne désignerait alors pas une fête spécifique); Plutarque, De Iside 29; Hésy-

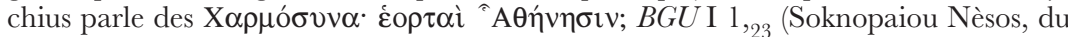
16 au 23 Phaophi au III ${ }^{\mathrm{e}}$ siècle apr. J.-C. = 13 au 20 octobre du calendrier julien); $B G U$ I 149, ${ }_{14}$ (II ${ }^{\mathrm{e}}-\mathrm{III}{ }^{\mathrm{e}}$ siècles apr. J.-C.); SPP XXII 183, ${ }_{68}$ (ca 138 apr. J.-C.); SB VI 9199, (milieu du II ${ }^{\mathrm{e}}$ siècle apr.J.-C.). Cf. également F. Perpillou-Thomas, Fêtes d'Égypte 75-76.

46 Cf. RICIS 501/0221.

${ }^{47}$ L'an 109 d'Auguste (i.e. de l'ère d'Actium) qui est aussi l'an 225 de l'ère macédonienne.

${ }_{48}$ RICIS 114/1901 = V. Gerassimova-Tomova, dans Lingu. Balk. 22.2 (1979) 31 $33 \mathrm{n}^{\circ} 1$ (non vidi) = ead., Zur Untersuchung einer antiken Stadt am mittleren Strymon (Struma), Klio 62 (1980) 19-26 = B. Sandanski, Musei i pametnici 20.2 (1980) 24-27 (non vidi).

49 V. Gerassimova-Tomova, Wirtschaftliche und religiöse Beziehungen Thrakiens zum Osten, Klio 62 (1980) 91-97. 95 est publié un autel (plutôt que «l'autel »?) du sanctuaire, consacré à Isitychè (= RICIS 114/1902). En outre, une plaque en marbre, anépigraphe, présentant deux empreintes de pieds de tailles différentes encadrées par deux sistres, provient du même sanctuaire. Il pourrait s'agir ici de l'empreinte du pied droit de la divinité apparue au (à la?) fidèle, représenté à côté de celui du (de la?) dédicant(e). 
habitants de la cité de Neine située sur le Strymon, quelque part entre Gradesnica et Ilindenci, certes encore en Macédoine, mais aux portes de la Thrace intérieure. Sous le principat de Marc Aurèle, au moins deux autres sanctuaires sont consacrés à Sarapis, l'un à Serdica, ${ }^{50}$ l'autre au Municipium Montanensium ou à Oescus ${ }^{51}$, tous deux attestés par l'épigraphie. La documentation numismatique permet d'allonger cette liste. Des temples renfermant une statue de Sarapis (debout ou trônant) figurent dans le monnayage d'Anchialos (sous Septime Sévère), d'Augusta Traiana (sous Septime Sévère, Caracalla et Gordien III), de Nicopolis ad Istrum (sous Caracalla, Élagabal et Gordien III), de Marcianopolis (sous Caracalla et Julia Domna, Macrin et Diaduménien, Gordien III et Tranquilline), d'Odessos et Tomis (sous Caracalla, Geta et Élagabal), enfin de Deultum (sous Gordien III) (figg. 2a-c). ${ }^{52}$ Cette situation semble liée au développement économique, culturel et urbain de la Mésie Inférieure et de la Thrace au $\mathrm{II}^{\mathrm{e}}$ siècle apr. J.-C. et durant la première moitié du III siècle. Au cour des cités, de nombreux espaces sacrés furent dédiés à

50 RICIS 114/1701 = S. N. Bobčev, Hram na Serapisa v Sofia [Serapistempel in Sofia], BIAB 14 (1940-1942) [1943] 218-222 = V. Beševliev, Epigrafski prinosi (Sofia 1952) $16-17$ n $^{\circ} 13=$ IGBulg IV $1926=$ SIRIS $134=$ B. Gerov, Prouchvaniya III $228 \mathrm{n}^{\circ}$ 38 = Tacheva-Hitova, Eastern Cults 20 n I.34. Le temple est dédié, en grec, à Zeus Capitolin Soleil Sérapis, Appius Claudius Martialis étant légat propréteur de Thrace.

51 RICIS 618/0102 = CIL III $12387=\mathrm{S}$. Frankfurter, AEM 14 (1891) $158 \mathrm{n}^{\circ} 6$ (cf. A. Stein, Die Legaten von Moesien [1940] 77, pour la date) $=$ SIRIS $702=$ TachevaHitova, Eastern Cults 3-4 n I.1. L'édification d'un temple avec statues pour Sarapis avait été entreprise par Marcus Iallius Bassus, légat d'Auguste propréteur, et achevée par l'un de ses successeurs, Pontius Laelianus peut-être. M. Iallius Bassus est connu par une autre inscription du Municipium Montanensium (CIL III 12373), dont pourrait alors provenir également la présente inscription, retrouvée cependant à Byala Slatina, à une trentaine de $\mathrm{km}$ à l'est de là. On ne peut toutefois pas écarter l'hypothèse d'une pierre provenant d'Oescus, sur le Danube, où l'on a déjà découvert une statue en pierre d'Isis ou d'une isiaque ainsi qu'un sistre en bronze; cf. Tacheva-Hitova, Eastern Cults 5 n I.3-4.

52 Sur ces monnaies, les façades représentées sont presque toujours celles de temples tétrastyles. On sait le peu de crédit qu'il faut toutefois accorder à ce type d'information à caractère architectural. Ceci étant, de tels types monétaires ne peuvent qu'attester l'existence d'un culte officiel de Sarapis dans les cités en question. Même opinion chez J.Jurukova, Religioznijat sinkretizăm Trakija, Mizija i Makedonija spored monetite ot rimskata imperatorska epocha, BIA 34 (1974) 41, B. Gerov, Marcianopolis im Lichte der historischen Angaben und der archäologischen, epigraphischen und numismatischen Materialien und Forschungen, dans: B. Gerov (éd.), Beiträge zur Geschichte der römischen Provinzen Moesien und Thrakien. Gesammelte Aufsätze (Amsterdam 1980) 294-295 (à propos de Marcianopolis), et U. Peter, SNRIS (à paraître). 


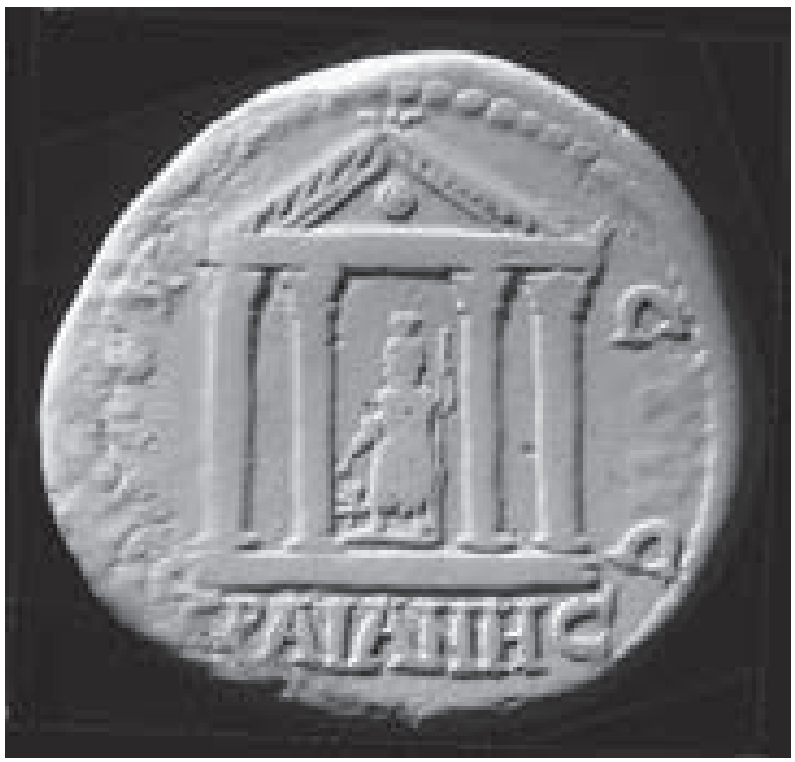

Fig. 2a. Monnaie d'Augusta Traiana. Règne de Caracalla (d'après E. SchönertGeiß, Augusta Traiana, 261).

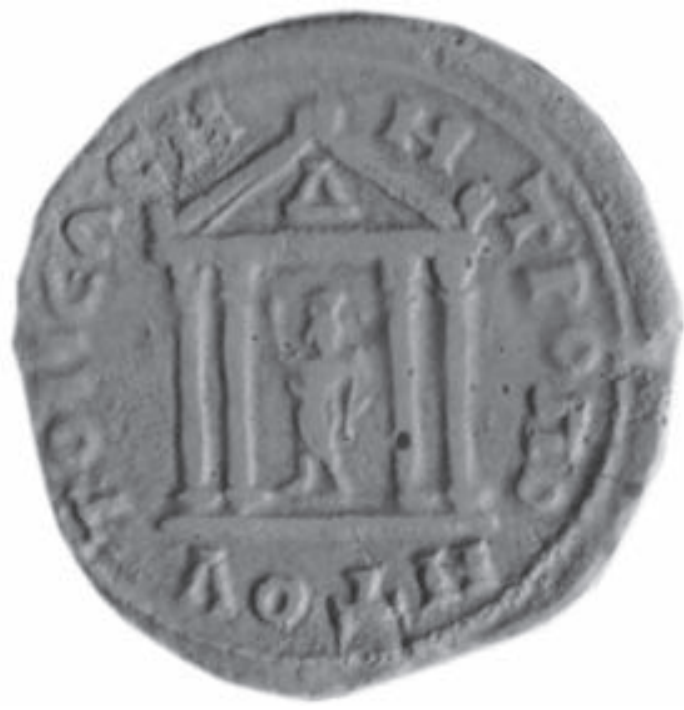

Fig. 2b. Monnaie de Tomis. Règne de Caracalla (d'après B. Pick, AMNG 2917). 


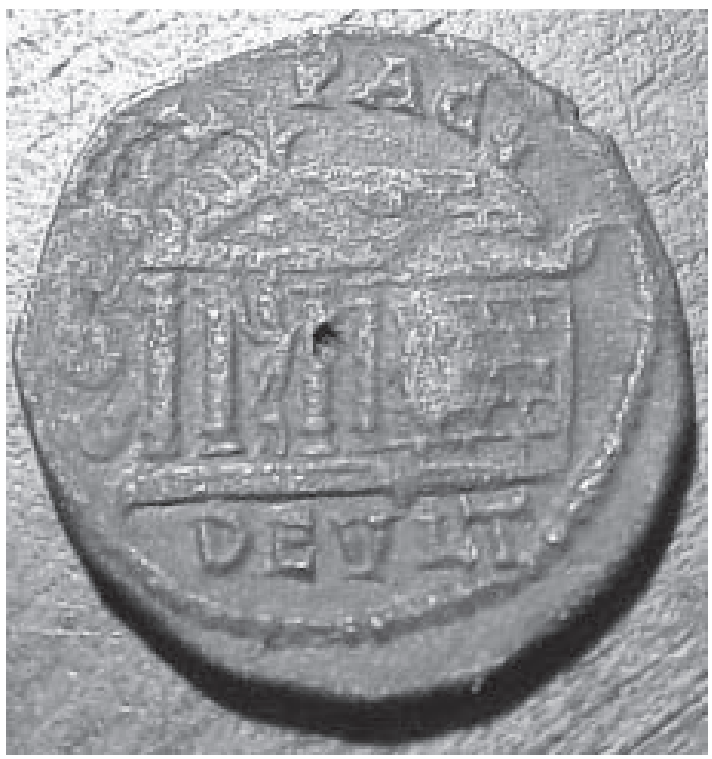

Fig. 2c. Monnaie de Deultum. Règne de Gordien III (Coll. privée).

Sarapis, certes, mais aussi et surtout, à Jupiter et à Asclépios, ${ }^{53}$ l'un et l'autre pouvant d'ailleurs être identifié au parèdre d'Isis comme à Byzance où Zeus Sérapis fut au moins une année, au Ir siècle av.J.C., le hiéromnémon, l'éponyme de la cité, ${ }^{54}$ à Serdica où, nous l'avons dit, un temple est dédié à Zeus Capitolin Sarapis sous Marc Aurèle, ou encore à Dionysopolis comme l'atteste un relief récemment publié portant un ex-voto à Zeus-Sérapis et Dionysos. ${ }^{55}$ Les processus d'identifications et de rapprochements divins qui se développent alors permettent sans aucun doute à Sarapis d'étendre son audience. Il est plus que probable qu'aux époques antonine tardive et sévérienne, le Théos Mégas d'Istros et d'Odessos a parfois été identifié à

${ }^{53}$ Cf. W. Szubert, Remarks on the Thracian-Roman Asklepieions, Études et Travaux XV (1990) 410-415, qui évoque des sanctuaires isiaques à Serdica et Deultum.

${ }^{54}$ RICIS 114/0701 = G. Mendel, Musées impériaux ottomans. Catalogue des sculptures grecques, romaines et byzantines III (Constantinople 1914) 42-43 n $838=\mathrm{L}$. Robert, Stèle de Byzance, Hellenica X (Paris 1955) 17-24 = SIRIS $129=$ I.Byzantion 19.

${ }^{55}$ I. Vasilčin, Monuments antiques des cultes orientaux sur la côte de la Mer Noire en Dobroudja, BMNVarna XXI (1985) 62-65. 
Sarapis, comme en témoignent de nombreuses monnaies. Avant le règne de Commode, le dieu représenté soit à cheval (à Istros), soit faisant une libation (à Odessos), l'est tête nue. À partir de la fin du $\mathrm{II}^{\mathrm{e}}$ siècle, il porte presque systématiquement le calathos (figg. 3a-b) ce qui, à mon sens, l'assimile dans ce cas à Sarapis, mais ne l'empêche pas par ailleurs de conserver son existence propre. ${ }^{56}$

Isis et les divinités de son cercle sont désormais indiscutablement présentes à Novae, ${ }^{57}$ Oescus,${ }^{58}$ Philippopolis ${ }^{59}$ et Ser-

56 Sur ce sujet discuté, cf. A. Suceveanu, Le «Grand Dieu» d'Histria, Ktèma 24 (1999) 271-281, et M. Oppermann, Überlegungen zum Kult des Theos Megas am Westpontos in römischer Zeit, Izvestija na Narodnïa Muzej Burgas 4 (= Studia in honorem Ivani Karayotov) (2002) 50-58. Le problème est entièrement repris dans la SNRIS par U. Peter, à partir d'une documentation très complète qui lui permet de parvenir à des conclusions claires et nuancées.

57 RICIS 618/0201 = ILNovae $24=$ IGLNovae 43. Dédicace de Valerius Eutychès à Sarapis sanctus, inscrite sur une base en calcaire brisée en haut, trouvée en 1989, qui fut remployée dans une pièce contiguë de la basilique chrétienne édifiée sur les ruines d'une ancienne villa. $\mathrm{II}^{\mathrm{e}}$ siècle apr. J.-C. Une seconde inscription pourrait concerner les cultes isiaques: RICIS $* 618 / 0202=\mathrm{L}$. Mrozewicz, Inskrypcje lacinskie, Archeologia 33 (1982) [1985] 196 (non vidi) = ILNovae 16 = IGLNovae 29. Elle est gravée sur un fragment de calcaire qui constitue le rebord gauche d'un grand bloc, trouvé en 1980 dans les déblais au centre de la nef principale de la basilique, dans le secteur ouest de Novae. Au début de la première ligne conservée, qui n'est pas la première de l'inscription, on lit les lettres ISI que les éditeurs songent à restituer en Isi di(?) - ]. La concavité du fragment a permis de calculer que l'ensemble du bloc devait mesurer au moins 1,70 m de large. La taille imposante des lettres peut laisser penser qu'il s'agit d'un fragment architectonique provenant d'un bâtiment, peut-être un Iseum pour les éditeurs. Ceci me semble bien hypothétique. $\mathrm{II}^{\mathrm{e}}$-III ${ }^{\mathrm{e}}$ siècles apr. J.-C. Enfin, Sarapis avait été reconnu par A. Sadurska, Fragmenty plastorzezby z postacia Sarapisa, Novae-Sektor Zachodni 1976 (1978) 191-195, sur un bas-relief découvert à Novae. L'identification du dieu ne me parait guère assurée.

${ }^{58}$ Cf. supra 255 n. 51.

59 RICIS 114/1501 = D. Cončev, Godisnik na Plovdivskata narodna biblioteka i muzej, 1937-1939 [1940] 155 n¹ $($ non vid $\imath)=$ IGBulg III, $1936=$ SIRIS $133=$ M. TachevaHitova, Eastern Cults, 23 n $^{\circ}$ I.37. L'inscription, dont le texte ne se déchiffre pas, mentionne cependant Sarapis. Le monnayage de la cité abonde en types isiaques. Particulièrement intéressant est le récipient cultuel découvert à Philippopolis et publié par M. Martinova, Ein antikes Kultgefäß aus Philippopolis, dans: M. Wendel (éd.), Karasura. Untersuchungen zur Geschichte und Kultur des alten Thrakien, I. 15 Fahre Ausgrabungen in Karasura (Weissbach 2001) 185-188 (notre fig. 4). Il est décoré, entre autres, de deux bustes de Sarapis au-dessus d'un aigle. M. Martinova, qui a bien noté l'existence du type sur les monnaies tomitaines, ne peut cependant affirmer qu'il s'agit d'une production locale et non d'un objet d'importation. Sur ce motif, cf. R. Veymiers, Sérapis et l'aigle: polysémie d'un iconotype, Acta Orientalia Belgica XVII (Louvain 2003) 267271. 


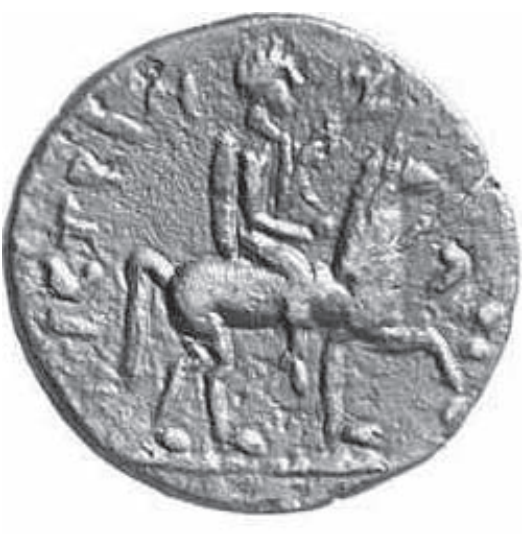

Fig. 3a. Monnaie d'Istros. Règne de Commode (Coll. privée).

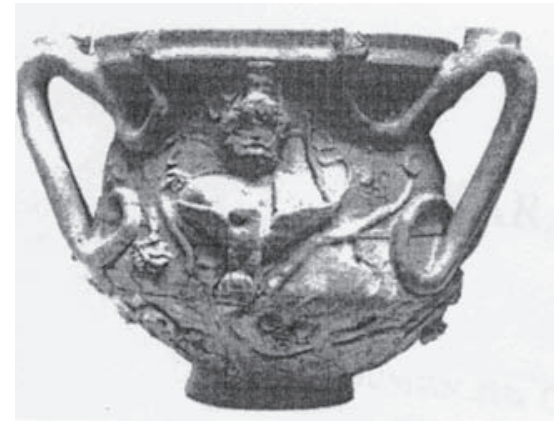

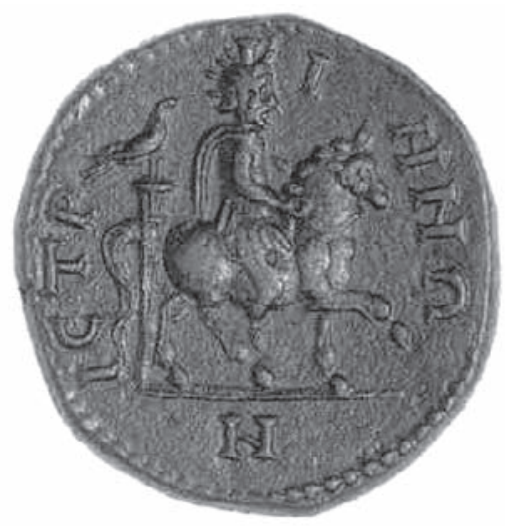

Fig. 3b. Monnaie d'Istros. Règne de Septime Sévère (Coll. privée).

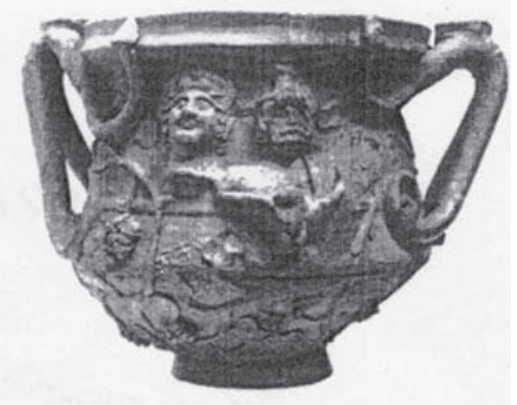

Fig. 4. Récipient de Philippopolis. (d'après M. Martinova, "Ein antikes Kultgefäß aus Philippopolis », dans M. Wendel (éd.), Karasura. Untersuchungen zur Geschichte und Kultur des alten Thrakien, I. 15 Jahre Ausgrabungen in Karasura (Weissbach, 2001), p. 186)

dica, ${ }^{60}$ voire au Municipium Montnensium. ${ }^{61}$ Elles le sont toujours dans l'île de Thasos, ${ }^{62}$ sur le littoral, à Maronée, ${ }^{63}$

60 À Serdica, on a recueilli une lampe en terre cuite aujourd'hui conservée au Musée de Sofia, qui porterait l'effigie de Sarapis. Cf. M. Stančeva, Kam izučavaneto na ezičeskite kultove v Serdika [Contribution à l'étude des cultes païens à Serdica], Musei i Pametnici na Kulturata 7.2 (1967) 6-10. L'identification du dieu ne me semble pas assurée.

\footnotetext{
61 Cf. supra 255 et n. 51.

62 RICIS 201/0102-0103.

63 Trois inscriptions attestent la pérennité du culte durant le Haut Empire.
} 
Périnthe, ${ }^{64}$ Byzance,${ }^{65}$ Tomis,${ }^{66}$ mais aussi à Abdère (?),${ }^{67}$ Cal-

Une dédicace de l'agonothète Apollônios s'adresse à Sérapis, Isis, Anubis et Harpocrate, sur leur ordre. Elle est gravée sur une base sur la face supérieure de laquelle on remarque les empreintes de deux paires de pieds orientées dans le même sens, mais de dimensions différentes, la paire droite plus longue que la gauche. Fin du $\mathrm{II}^{\mathrm{e}}$-début du III ${ }^{\mathrm{e}}$ siècle apr. J.-C. RICIS 1 14/0204 = Y. Grandjean, Maronée 119-120 = TachevaHitova, Eastern Cults 31-32 n I.52 = Dunbabin, Ipsa deae vestigia 87 fig. $2=$ D. Triantaphyllos, dans: $\Theta \rho \alpha \kappa \imath \kappa \eta \dot{~ E \pi \varepsilon \tau \eta \rho i ́ \delta \alpha ~} 10$ (1995-1998) ph. 363 (non vidi).

Deux autres dédicaces, probablement de même époque, s'adressent elles aussi à la tétrade: RICIS 114/0205 = D. Triantaphyllos, AD 27 B2 (1972) [1977] 540, et RICIS 114/0206 = D. Triantaphyllos, AD 48 B2 (1993) [1998] 405.

${ }^{64}$ Pendant plusieurs siècles, de nombreuses émissions locales figurent Isis et Sarapis, mais aussi Anubis, Apis et Harpocrate. Un sanctuaire des divinités isiaques a dû exister durant toute la période. Cf. E. Schönert, Die Münzprägung von Perinthos (Berlin 1965) IS n 31-58, 92, 149-156, 224-226, 259-271, 602-606, 749, 782-783, 860 et 896; voir 54-55 sur la continuité du culte du III' siècle av. J.-C. au III' siècle apr. J.C. La question est reprise par U. Peter dans la SNRIS. Cf. également l'amulette en or trouvée dans un sarcophage exhumé devant le mur occidental de la ville et portant

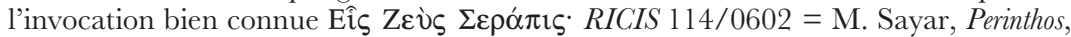
$226 \mathrm{n}^{\circ} 43$ et pl. XV, figg. 57-58. I $^{\text {er-II }}{ }^{\mathrm{e}}$ siècles apr. J.-C.

${ }^{65} \mathrm{Au}$ moins une sinon deux stèles funéraires d'époque impériale portent des représentations de femmes qui ont pu être des isiaques. Sur la première, fragmentaire, découverte à Istanbul (Beyazit), on trouve une femme, vêtue du chiton et de l'himation noué sur la poitrine. Ce nœud, ainsi que la représentation d'une ciste suggèrent qu'il s'agit d'une isiaque, dont le nom ne peut se déchiffrer: RICIS 114/0704 = N. Firatli, Les stèles funéraires de Byzance gréco-romaine, avec l'édition et l'index commenté des épitaphes, par L. Robert (Paris 1964) $100 \mathrm{n}^{\circ} 150=$ I.Byzantion 138. Une seconde stèle funéraire, aujourd'hui égarée, au nom de Glykea, fille d'Hérakôn, est republiée par A. Latjar, I.Byzantion 139. Découverte en 1835 lors de l'édification de l'église arménienne de l'Archange Michel, elle fut décrite sommairement par K. Lehmann, Byz.-ngr. Fahrbuch 1 (1920) 381 n. 2, qui notait le vêtement à franges qu'elle portait. Le dernier éditeur songe à en faire une isiaque, ce qui est possible mais non assuré en l'absence de tout support iconographique.

Quant à la stèle de marbre représentant un banquet funéraire et portant une première inscription, au-dessus du relief, martelée pour faire place à l'épitaphe de Poseidonios, fils d'Artémidôros, elle présente sur la marge inférieure une autre inscription, dédicace à Isis d'une certaine Épikratè, fille de Dionysios, addition postérieure faite lors du second remploi de la stèle: RICIS 114/0702 = N. Firatli, Stèles funéraires de Byzance 59 n $^{\circ} 41$ = G. Daux, BCH 91 (1967) 480-481 = Cl. Vatin, La stèle funéraire de Byzance $\mathrm{n}^{\circ} 41$, BCH 92 (1968) 220-225, avec un appendice de Ph. Bruneau = SIRIS $129 \mathrm{a}=$ I.Byzantion 16. La scène du banquet funéraire a pu être réutilisée sans difficulté dans un contexte isiaque, le banquet devenant alors la représentation figurée de la $\kappa \lambda i ́ v \eta$ d'Isis.

${ }^{66}$ RICIS 618/1004-1007.

67 RICIS 114/0101. Simplement mentionnée par Ch. Koukouli, AD 24 (1969) Chron.356, l'inscription, une dédicace à Isis et à Sarapis par un certain Diês, fils d'Euphémos, a été publiée de manière incomplète par D. Hereward, An inscription from Thrace, ZPE 17 (1975) 125-126 (Bull. 1976, 463), et, indépendamment, par 
latis, ${ }^{68}$ Troesmis ${ }^{69}$ et Olbia, où un temple avec portique est édifié aux dieux qui écoutent les prières Sarapis, Isis, Asclépios, Hygie et Poséidon, pour la fortune de Sévère Alexandre, du saint Sénat et des troupes ainsi que pour le salut et la stabilité de la cité. ${ }^{70}$ Toutefois, aucun sanctuaire isiaque de Mésie Inférieure et de Thrace n'a jusqu'à présent été découvert. Cependant, le témoignage des monnaies, plus que des inscriptions, fait d'Odessos ${ }^{71}$ Marcianopolis, Mesembria, ${ }^{72}$ Nicopolis ad Istrum, ${ }^{73}$ Bizye, Deultum, Augusta Traiana, Hadrianopolis, Traianopolis, Pautalia des cités où les cultes isiaques sont bien implantés à l'époque sévérienne, et même encore au milieu du IIIe siècle, Gordien III et Philippe II étant régulièrement associés à Sarapis au droit des pièces (figg. 5a-b). ${ }^{74}$ Le dieu, comes Augusti, prend alors incontestablement le pas sur Isis, qui n'apparaît plus

Tacheva-Hitova, Eastern Cults 32 n I.53 et pl. XVI (qui ne connaît pas les publications antérieures, et dont l'édition n'est guère satisfaisante). L'autel en marbre blanc, très détérioré, sur lequel a été gravé l'inscription, provient d'une église St Georges à Mandra, aurait par la suite été transporté à Xanthe et est actuellement conservé au Musée de Cavalla, $\mathrm{n}^{\circ}$ inv. $\Lambda$ 784. La provenance abdéritaine n'est donc pas assurée. $\mathrm{II}^{\mathrm{e}}$ siècle apr. J.-G.?

68 Stèle funéraire de la fille d'un isiaque. RICIS 618/0901 = A. Avram, Un nouveau document sur le culte d'Isis à Callatis à l'époque impériale, RRÉ 1 (1997) 5-11 $=i d$., Inscriptions grecques et latines de Scythie Mineure. III, Callatis et son territoire (Bucarest et Paris 1999) 505-507 n 183. Fin $\mathrm{II}^{\mathrm{e}}$-début $\mathrm{III}^{\mathrm{e}}$ siècles apr. J.-C.

69 RICIS 618/1201 = CIL III $6164=$ SIRIS $710=$ Tacheva-Hitova, Eastern Cults $6 \mathrm{n}^{\circ} \mathrm{I} .7=$ ISM V $205 \mathrm{n}^{\circ} 168=\mathrm{L}$. Bricault, Notes à propos de CIL III 6164, ZPE 91 (1992) 215-216. Cette dédicace en latin à IOM Sérapis, gravée sur le chapiteau d'un pilastre en calcaire, est sans doute à mettre en relation avec la présence dans la ville, aux II ${ }^{\mathrm{e}}$-III ${ }^{\mathrm{e}}$ siècles apr. J.-C., de la legio V Macedonica.

70 RICIS 115/0201 = IOSPE $\mathrm{I}^{2} 184=$ SIRIS 713.

71 Rappelons toutefois la découverte de plusieurs statuettes en bronze dans les thermes d'Odessos et, parmi celles-ci, d'une statuette d'Isis-Fortuna (h. 14,5 cm) aujourd'hui conservée au musée de Varna ( $n^{\circ}$ inv. II 5305) (notre fig. 6). Cf.Georgiev, Plastique en bronze des Thermes romains à Varna, Arch (Sofia) 20 (1978) 33-39 (en bulgare, résumé en français 39), 34 fig. 4 pour Isis. Il n'est pas possible de certifier qu'il s'agit d'un travail local, quoique l'auteur le suggère fortement.

72 I. Karajotov, Mesambrijskijat panteon, dans: Fubilaeus V: In honorem Prof. Dr. Margaritae Tacheva (Sofia, 2002), 206.

${ }^{73}$ C'est peut-être de Nicopolis que provient en réalité une plaque en marbre trouvée en 1959 à Butovo-Nedan, située à quelques km à l'ouest de l'actuelle Nikyup, présentant en relief Diane chasseresse (notre fig. 7), dédiée par un certain Iulianus Taurus, prêtre d'Isis: RICIS 618/0301 = B. Gerov, Inscriptiones Latinae in Bulgaria repertae. Inscriptiones inter Oescum et Iatrum repertae (Sofia 1989) n 440.

${ }^{74}$ En attendant la publication de la SNRIS, on pourra se reporter aux tableaux de synthèse réalisés par Tran tam Tinh, Sérapis et Isis en Thrace et en Mésie Inférieure: problèmes iconographiques, dans: Pulpudeva, Semaines philippopolitaines de l'histoire et de la culture thraces 6-Supplementum, Plovdiv, 10-12 octobre 1986 (Sofia 1998) 172-195. 


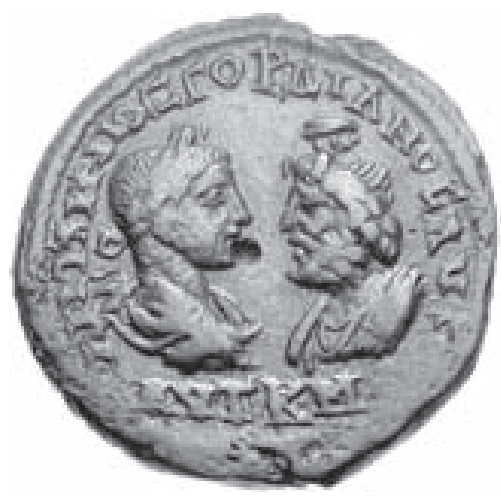

Fig. 5a. Monnaie de Marcianopolis. Règne de Gordien III (Coll. privée).

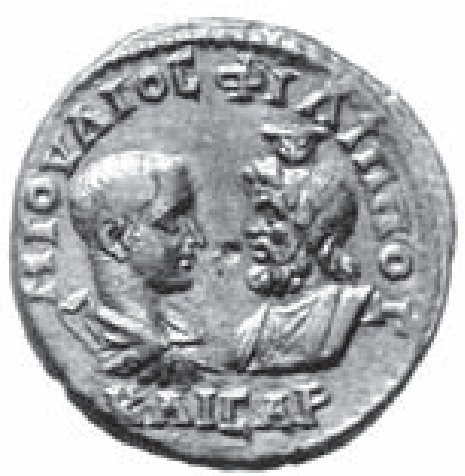

Fig. 5b. Monnaie de Tomis. Règne de Philippe Ier (Coll. privée).

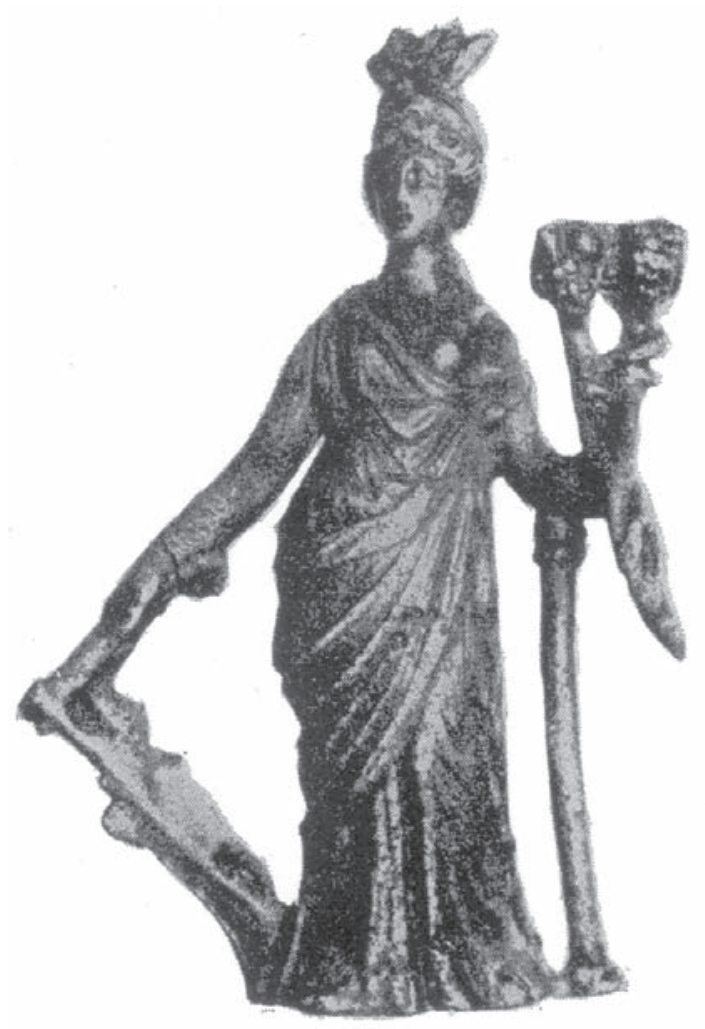

Fig. 6. Statuette d'Isis-Fortuna d'Odessos (d'après P. Georgiev, « Plastique en bronze des Thermes romains à Varna ", Arch (Sofia) 20 (1978), p. 34 fig. 4). 


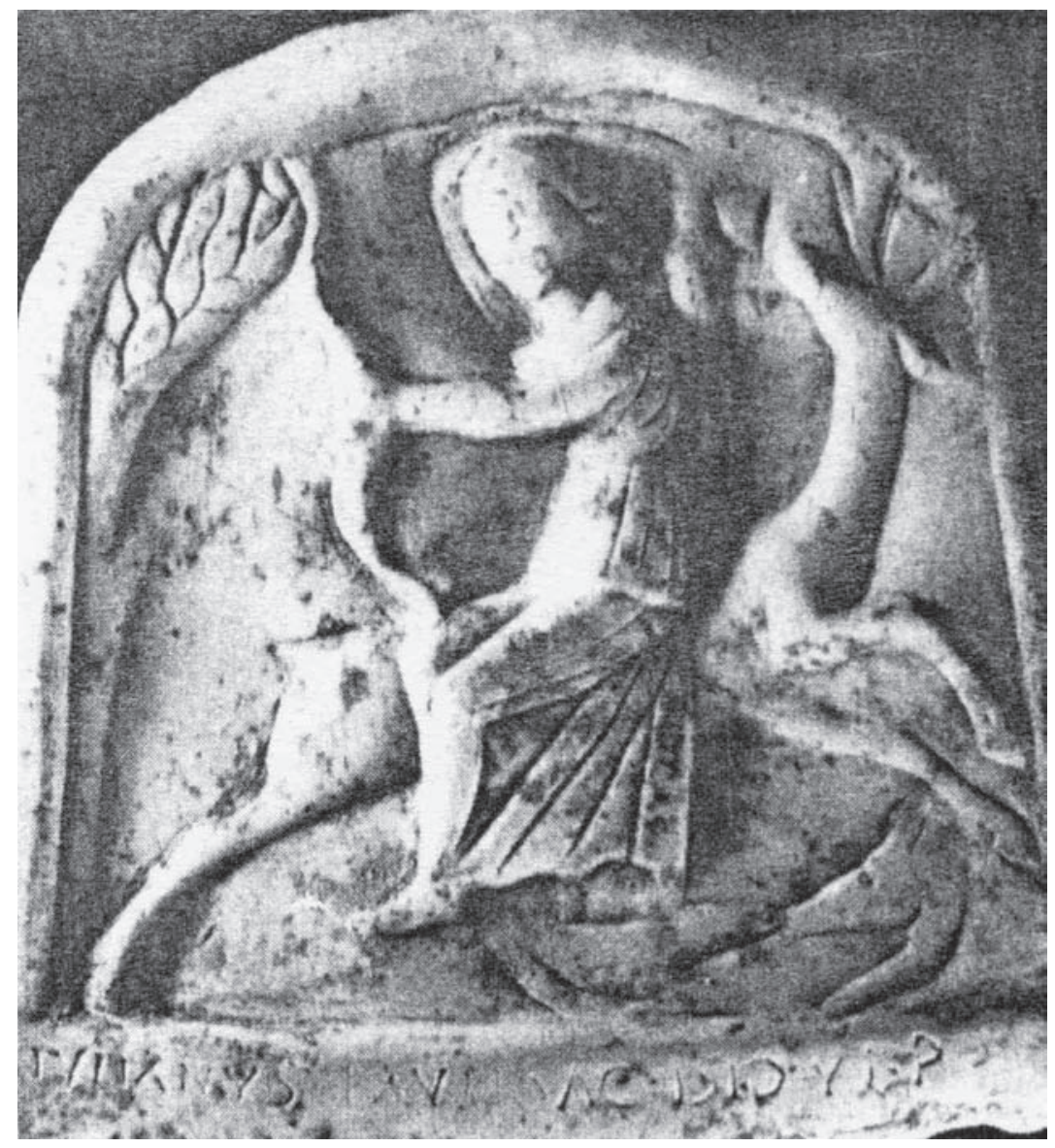

Fig. 7. Dédicace d'un prêtre d'Isis, Butovo-Nedan (d'après B. Gerov, Inscriptiones Latinae in Bulgaria repertae. Inscriptiones inter Oescum et Iatrum repertae (Sofia, 1989), $\mathrm{n}^{\circ}$ $440)$.

que sporadiquement dans la documentation, essentiellement sous l'aspect d'Isis Pelagia, patronne de la navigation. Des monnaies au type d'Isis à la voile sont frappées à Callatis sous Commode, Septime Sévère et Gordien III ${ }^{75}$, à Philippopolis sous Commode (fig. $8 \mathrm{a})^{76}$, à Byzance sous Caracalla ${ }^{77}$, à Anchialos sur des monnaies

\footnotetext{
75 SNRIS Callatis 2, 4 et 8.

76 SNRIS Philippopolis 4.

77 SNRIS Byzantium 1.
} 


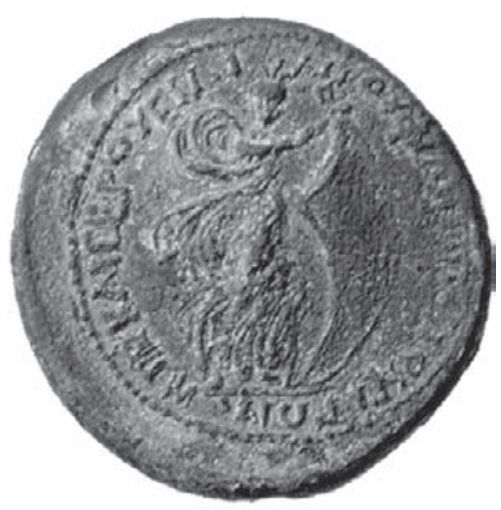

Fig. 8a. Monnaie de Philippopolis. Règne de Commode (d'après le catalogue de la maison G. Hirsch Nachf. 203 (1999), 601).

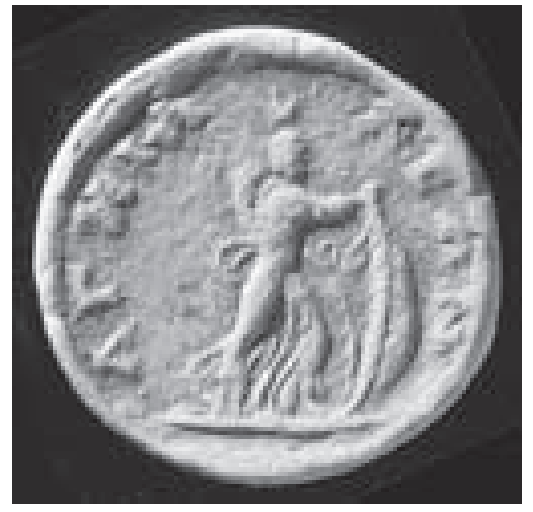

Fig. 8b. Monnaie d'Anchialos. Début IIIe s. apr. J.-C. (d'après B. Pick, $A M N G$, 408).

d'époque sévérienne présentant le buste de Sarapis au droit (fig. 8b) ${ }^{78}$ enfin à Périnthe sous Sévère Alexandre. ${ }^{79}$ Si la présence, à l'époque impériale, d'un culte rendu à Isis marine dans les ports de Callatis, Anchialos et Byzance ne peut surprendre, les émissions de Philippopolis et de Périnthe méritent un commentaire. Philippopolis, en Thrace intérieure, est située à près de $200 \mathrm{~km}$ de la mer de Thrace, mais dispose d'un port fluvial sur le fleuve Hébros, ce qui invite à penser qu'Isis pouvait apparaître comme maitresse de la navigation fluviale, à l'instar de Sarapis. ${ }^{80}$ Quant à l'émission de Périnthe (fig. 9), elle présente un motif plus complexe, qui concerne la présence isiaque dans la cité aussi bien directement qu'indirectement. Sur le pont d'une galère navigant à gauche, on trouve à la proue Isis Pelagia debout à gauche, la tête de face, tenant une voile de ses deux mains, dans le sens de la marche du navire; au milieu, Sévère Alexandre, vêtu de la toge, debout de face, la tête à gauche,

78 SNRIS Anchialus 15.

79 SNRIS Perinthus 14.

80 Sur ce point, on verra nos remarques dans Isis, Dame des flots (à paraître), et celles présentées par U. Peter lors du XIII Congreso Internacional de Numismática de Madrid en 2003, sur la relation existant entre ce type et la navigation sur l'Hébros. Pour Sarapis protecteur de la navigation fluviale, cf. déjà L. Bricault, Présence isiaque dans le monnayage impérial romain, dans: F. Lecocq (éd.), L'Égypte à Rome. Actes du colloque international de Caen 28-30.11.2002, Cahier de la MRSH-Caen nº 41 103-104. 


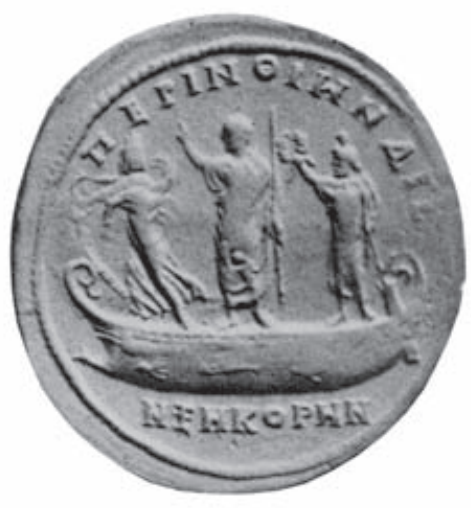

Fig. 9. Monnaie de Périnthe. 231 apr. J.-C. (d'après le catalogue de la maison Freeman \& Sear 5 (1999), 110)

salue de la droite levée et tient un sceptre de la gauche; à la poupe, Sarapis, debout de face, la tête coiffée du calathos tournée à gauche, vêtu d'un long pallium, couronne l'empereur de la droite, la gauche tenant le gouvernail du bateau. Cette émission doit dater de 231 apr. J.-C. et commémorer l'embarquement, depuis Périnthe, de Sévère Alexandre parti affronter les Sassanides. L'expédition, à commencer par la traversée, est ainsi placée sous la protection d'Isis et de Sarapis, divinités adorées de longue date dans la cité thrace et garantes d'une victoire qui ne saurait échapper aux Romains. ${ }^{81}$

Comme on vient de le voir, les cultes isiaques, au milieu du III ${ }^{\mathrm{e}}$ siècle après J.-C. et sans doute plus tardivement,- Sarapis figure encore sur des médaillons de Périnthe frappés sous le règne de Gallien - ${ }^{82}$ sont bien présents dans les centres urbains du territoire méso-thrace. Telle n'est cependant pas la seule aire de diffusion de ces cultes. De nombreux petits objets, à commencer par des statuettes, ont été retrouvés en des lieux divers, cités de second rang et villae romaines. ${ }^{83}$ En l'absence de fouilles spécifiques et scientifiquement fiables, on doit la plupart du temps se contenter de mentionner ces

\footnotetext{
${ }^{81}$ Cf. E. Schönert-Geiss, Perinthos 43.

82 SNRIS Perinthus 16.

83 Cf. par exemple I. Mitrofan, Sérapis sur un moule de l'habitat romain de Mičasasa, SCIVA 43 (1992) 55-61 (en roumain).
} 
objets sans pouvoir leur attribuer une provenance précise, ni leur donner une signification autre que générale. ${ }^{84}$

Il semble donc que la diffusion des cultes isiaques en Thrace et en Mésie Inférieure se soit opérée dès le $\mathrm{III}^{\mathrm{e}}$ siècle av. J.-C., d'abord, comme il se doit, sur les littoraux, puis plus avant vers l'intérieur à partir de l'époque flavienne. Les dernières traces cultuelles se perdent dans les soubresauts de la fin du III ${ }^{\mathrm{e}}$ siècle apr. J.-C. Isis et Sarapis paraissent avoir suivi dans ces régions, des voies parfois aussi divergentes que convergentes. Isis, assimilée d'abord à Aphrodite et à Arsinoé II, y est vénérée comme divinité protectrice des marins, un rôle dont la popularité ne se dément pas six siècles durant, y compris sur le réseau fluvial. Elle est aussi une divinité guérisseuse, à l'instar de son parèdre qui, quant à lui, semble avoir eu quelque difficulté à s'implanter, avant de connaître le succès, certes relatif, aux époques antonine et sévérienne, bénéficiant de l'appui du pouvoir impérial, mais aussi d'un rapprochement qui n'est peut-être pas fortuit avec le Théos Mégas.

84 Aux documents réunis dans l'Atlas, outre la statuette de Madara évoquée supra n. 36 et le récipient de Philippopolis mentionné n. 59, on ajoutera:

une statuette d'Isis en bronze provenant de Bizone, dans la Dobroudja; cf. I. Vasilčin, Monuments antiques des cultes orientaux sur la côte de la Mer Noire en Dobroudja, BMNVarna XXI (1985) 62-65;

un buste en bronze de Sarapis provenant de Bukovets, conservé au Musée de Veliko Tarnovo; cf. M.-Chr. Budischovsky, dans: Isis en Occident, 174.

une statuette en bronze d'Harpocrate provenant de Tchomakovtzi, conservée au Musée archéologique de Sofia, ${ }^{\circ}$ inv. 6073; cf. Le Bronze sculpté de l'époque romaine au Musée archéologique National de Sofia (1984) nº 186. 